\title{
Change Detection Based Building Damage Assessment Method Using Radar Imageries with GLCM Textural Parameters
}

\author{
Asset Akhmadiya ${ }^{1,2}$, Nabi Nabiyev ${ }^{2}$, Khuralay Moldamurat ${ }^{2}$, Kanagat Dyusekeev ${ }^{1}$, Sabyrzhan Atanov ${ }^{1}$ \\ ${ }^{1}$ Eurasian National University, Nur-Sultan, Kazakhstan \\ ${ }^{2}$ S.Seifullin Kazakh Agro Technical University, Nur-Sultan, Kazakhstan \\ Corresponding: aaset-04-77@mail.ru. ORCID:0000-0001-9136-7999
}

\begin{abstract}
In this research paper, change detection based methods were considered to find collapsed and intact buildings using radar remote sensing data or radar imageries. Main task of this research paper is collection of most relevant scientific research in field of building damage assessment using radar remote sensing data. Several methods are selected and presented as best methods in present time, there are methods with using interferometric coherence, backscattering coefficients in different spatial resolution. In conclusion, methods are given in end, which show, which methods and radar remote sensing data give more accuracy and more available for building damage assessment. Low resolution Sentinel-1A/B radar remote sensing data are recomended as free available for monitoring of destruction degree in microdistrict level. Change detection and texture based method are used together to increase overall accuracy. Homogeneity and Dissimilarity GLCM texture parameters found as better for separation of a collapsed and intact buildings. Dual polarization $(\mathrm{VV}, \mathrm{VH})$ backscattering coefficients and coherence coefficients (before earthquake and coseismic) were fully utilized for this study. There were defined the better multi variable for supervised classification of none building, damaged and intact buildings features in urban areas. In this work, we were achieved overall accuracy 0.77 , producer's accuracy for none building is 0.84 , for damaged building case 0.85 , for intact building 0.64 . Amatrice town was chosen as most damaged from 2016 Central Italy Earthquake.
\end{abstract}

Keywords: radar remote sensing; building damage assessment; change detection method; GLCM

\section{INTRODUCTION}

Natural disasters are occurred accidentally and unpredictable. They create huge amount of losses which are concentrated mainly in cities, in places where it is more densely population. It is crucial to rescue and to start the recovery work after any natural disaster, which must be done immediately. It is vital to have operational map of building destruction for a rapid and efficient planning of rescue operations from the rubble of the building and to reduce the death rates. Moreover, this information is necessary for damage assessment of natural disasters in the field of urban planning and recovery works.

Natural disasters are often accompanied by rain, clouds and other adverse weather conditions which affected on optical remote sensing data quality and applicability. Radar remote sensing data is devoid of such disadvantages, it can be widely used in respect of its allweather and day-night operation, quick access to disaster, continuous dynamic monitoring over affected territory, it has becomes an important means for disaster assessment. Since 2000, European Space Agency (ESA) and French Space Agency (CNES) became initiators of the agreement about International Charter on Space and Major Disasters, which includes charitable use of images acquired from different satellites of radar remote sensing, these data is important data source.

Radar remote sensing data with their interferometric coherence coefficients have been first used to monitor damage caused by surface deformation, it was the starting point of the research. These data had been low and midlle spatial resolution with one or two polarizations. Before and after earthquake radar remote sensing data were used for building damage assessment purposes in urban area, their backscattering, intensity correlation and coherence coefficients [1-4].

Starting in 2007, TerraSAR-X, Radarsat-2, COSMOSkyMed, TANDEM-X satellites were launched, which had 2-4 polarizations, and the spatial resolution of image was up to 1 meter. It was possible to see in details city structures or individual buildings in such spatial resolution $[1,4,5]$. These satellite data allow us to identify of building, because each building has unique backscattering characteristic and signature (visible shape) under certain regular conditions, any significant changes can say that there have damaged buildings or homes.

Building damage assessments methods using radar remote sensing data is relevant, many scientists try to get more accurate methods or algorithms for extraction of damaged buildings or creation damage map. Characteristics of collapsed and intact buildings in radar remote sensing data should be considered for that purposes. In addition, higher accuracy change detection based building damage assessment methods should be defined, which methods out of them are accurate and reliable.

\section{CHANGE DETECTION METHOD}

In the context of remote sensing, change detection refers to the process of identifying differences in the state of land features by observing them at different times. Discriminating areas of changes on radar imageries between different dates is the main objective of change detection process. Change detection method can be applicable to assess damage in urban areas for natural disaster for that purposes, especially earthquakes. Unlike of optical, where intensity is sensitive to chemical 
properties of surface and roughness, for radar, reflection and scattering totally depends on geometrical properties of surface, especially in urban areas, where backscattering intensity depends on different geometric shape of buildings and distance between and etc. The use of radar imageries for detecting change on the earth's surface is highly dependent on target orientation, azimuth angle, and sensor depression angle [6,37]. Because of changes in look direction is as small as $0.8^{\circ}$ between the orientation of building and the differences in the radar azimuth can cause major changes in the radar backscatter, which affect the capability to detect the changes in urban areas [7]. Another important aspect related to depression angle is affecting the use of radar imageries for change detection method. For aircraft radar it is more important than space borne radar, because it varies extensively from near nadir. There should be such rules in space borne radar systems: a) gathering data using a constant bearing for any geographical location (assuming ascending or descending passes), b) gathering data over an long period of time, c) assemble data at fixed wavelength and polarization, d) gathering data with a small depression angles $\left(6^{\circ}\right)$. The use radar data sets separated only in time is better than those separated in time and look direction for change detection studies [8]. In change detection method, major concern is speckle noise, it is reported due to speckles in the images which cannot be avoided results in uncertainty and decrease value of the image, hence application of the image automatically becomes very difficult. It is problem for low resolution radar imageries, when size of building is comparable with the pixel resolution. For high resolution SAR imagery, when building is clearly visible, its edges, like rectangular shape, here it is possible to use the smoothing algorithms based on local statistics [9].

In general, in monitoring disasters by using pre and post-earthquake radar imageries had been found problems $[10,11]$. Due to earthquakes in backscattering coefficient occur the change of correlation and coherence for damaged buildings. Since, building is generally relatively stable construction, if the coherence changed, it can deduce that building is damaged. Based on this changing from aforementioned reference, methods can be divided into two kinds. One is the use of pre and post-earthquake data to generate coherence coefficient of InSAR product for damage detection. Coherence based change detection method is used for building damage detection via decoherence. Second is the use of also multi-temporal radar data to generate intensity correlation coefficient for damage detection. Intensity correlation based change detection method is used for building damage detection via decorrelation.

Since 1998, scientists from Japan began to use the intensity correlation coefficients for detection of damaged buildings, for that purposes were used to ERS-1 data. Intensity correlation coefficient of SAR imageries can give better result under certain condition for determination of earthquake zones. Yonezawa et al. [12] offer to use interferometric coherence coefficient for detection of damaged buildings in urban areas, there have been used pre and post-earthquake ERS-1 data for 1995 year, Kobe,
Japan. It was discovered that coherence coefficient in the damaged part of the city less than intact. In addition, Matsuoka et al. [13] using ERS-1/2, Hosokawa [14] using JERS-1 data carried out the same research. However, in practice the use of interferometric coherence has some limitations, it is depends of many parameters (baseline length, time interval, wavelength, surface change, seasonal vegetation changes and etc.), which affect on loss of coherence, therefore, the choice of paired radar data should be rigorous with taking into account the cause of these losses. By combining these methods it is possible to achieve efficient detection of damaged buildings. Yonezawa et al. $[15,16]$ have considered two methods in their research based on coherence and intensity correlation for damaged buildings and the extent their relationship. Relationship intensity correlation and coherence before and after earthquake under different baseline distances for damaged and undamaged urban areas was considered in these research. Both of the correlations are significantly influenced by the baseline distance. The short baseline distance data pairs indicated clear decorrelation in the damaged area than long. However, it is difficult to distinguish damaged and undamaged areas by long baseline distance data pairs, because it leads to decorrelation in whole areas, the greater the distance the worse it is to distinguish them. The degree of decorrelation indicated by the normalized difference of two correlation coefficients increases with the proportion of damaged buildings in the area.

How to calculate those aforementioned correlation coefficients and degree of decorrelation? The norm type correlation coefficient of the two single-look intensity images (intensity correlation) in a small corresponding patch is calculated as follows:

$$
\rho_{I}=\frac{\left|E\left(I_{1} I_{2}\right)\right|}{\left[E\left(I_{1}^{2}\right) E\left(I_{2}^{2}\right)\right]^{1 / 2}}
$$

where $I_{1}$ and $I_{2}$ are corresponding pixel values of the two single-look intensity data, $E()$ denotes statistical expectation.

The coherence is calculated as complex correlation coefficient $\rho_{c}$ in a small corresponding patch as follows:

$$
\rho_{c}=\frac{\left|E\left(c_{1} c_{2}^{*}\right)\right|}{\left|E\left(c_{1} c_{1}^{*}\right) E\left(c_{2} c_{2}^{*}\right)\right|^{1 / 2}}
$$

where $c_{1}$ and $c_{2}$ are the corresponding complex values of the two SLC data, $c_{i}^{*}$ means the complex conjugate of $c_{i}$.

Two intensity correlation coefficients can be used for calculation of a normalized difference $N d$ :

$$
N d_{i}=\left[\frac{\rho_{I_{1}}-\rho_{I_{2}}}{\rho_{I_{1}}+\rho_{I_{2}}}\right] \times G n+O f
$$

where $\rho_{I_{1}}$ is intensity correlation correlation coefficient, which obtained before earthquake, $\rho_{I_{2}}$ is intensity correlation coefficient from the data pair whose interval included the earthquake occurrence, $G n$ is gain and $O f$ is offset.

Matsuoka et al. have considered the effects of speckle noise reduction and pixel window size in evaluating building damage using the difference in the backscattering coefficient and correlation coefficient of the pre- and postevent radar imageries. There were used ERS data [3], the 
statistical relationships were presented for classification of damage level, there achieved good results in the Kobe earthquake. In further, they were used with Envisat-ASAR for detection of damaged urban areas due to the Bam Earthquake in Iran [17]. Additionally, this model was improved for ALOS-PALSAR L-band, same as that which was used for the 1995 Kobe earthquake, it was used for the 2007 Peru earthquake [18]. There were used the backscattering intensity difference, intensity correlation and discriminant scores for detection of damaged urban areas, they also gave good results. Hosokawa [14] had also conducted relevant researches using JERS-1.

Japanese scientist Matsuoka have proposed to use the difference of backscattered intensity in $\mathrm{dB}$, correlation coefficient of intensity, degree of complex coherence by using pre- and post-earthquake ERS data for different damaged rank A, B, C, D, E, which represents in percentage ratio $0-6.25,6.25-12.5,12.5-25,25-50$, $50-100 \%$.

Correlation and difference in the backscattering coefficient of the two basic value, which were used as the indices representing the changes in affected areas. The correlation coefficient, $r$, between two intensity images, $a$ and $b$, is calculated within a small corresponding window as follows [3]:

$$
r=\frac{N \sum_{i=1}^{N} I_{a_{i}} I_{b_{i}}-\sum_{i=1}^{N} I_{a_{i}} \sum_{i=1}^{N} I_{b_{i}}}{\sqrt{\left(N \sum_{i=1}^{N} I_{a_{i}}^{2}-\left(\sum_{i=1}^{N} I_{a_{i}}\right)^{2}\right)\left(N \sum_{i=1}^{N} I_{b_{i}}^{2}-\left(\sum_{i=1}^{N} I_{b_{i}}\right)^{2}\right)}}
$$

where $i$ is the sample number, $I_{a_{i}}$ and $I_{b_{i}}$ are the digital numbers of the post- and pre-images, $N$ is total number of pixel in window size. The difference of backscattered is calculated as follows:

$$
d=10 \log _{10} \bar{I}_{a_{i}}-10 \log _{10} \bar{I}_{b_{i}}
$$

where $\bar{I}_{a_{i}}$ and $\bar{I}_{b_{i}}$ are the corresponding averaged digital numbers over the surroundings of pixel $i$ within a window size.

Next questions are follows which values of the correlation and difference in the backscattering coefficient belong to damaged and undamaged urban areas, the distribution of these values in two-dimensional plot. Matsuoka et al. offer to use a linear discriminant line which used to classify these areas. Here, they introduced a new value called as discriminant score $z$.

Interferometric coherence also useful tools for detection of damaged building. Many scientist tried to use coherence coefficient before and after earthquake, it gives good results, if baseline shorter than critical baseline distance [19] and more longer wavelength [20]. Value of interferometric coherence coefficient $|\gamma|$ depends on many factor, it consists of multiply parts, each part can cause loss of coherence [21]:

$$
|\gamma|=\gamma_{\text {thermal }} \gamma_{\text {misreg }} \gamma_{\text {spat }} \gamma_{\text {dopp }} \gamma_{\text {atm }} \gamma_{\text {temp }}
$$

where $\gamma_{\text {thermal }}$ caused by uncorrelated noise inside the radar sensor itself, $\gamma_{\text {misreg }}$ is due to inaccurate registration of the two radar imageries, $\gamma_{\text {spat }}$ depends of large baseline distance [22], it should be less than critical baseline distance $B_{\text {critical }}, \gamma_{\text {dopp }}$ doppler centroid de-correlation, which mitigates by using the range adaptive azimuth common band filtering, $\gamma_{a t m}$ is due to different atmospheric conditions, may cause artifacts in the interferogram, $\gamma_{\text {temp }}$ - caused by changes on the ground in the time between radar data acquisition dates.

The most important are $\gamma_{\text {spat }}$ and $\gamma_{\text {temp }}$, others can be accounted. For each of pair SAR data should be selected baseline distance less critical $B_{\text {critical }}$, which is calculated as follows:

$$
B_{\text {critical }}=\frac{\lambda R_{s}}{2 r(\cos \theta)^{2}}
$$

where $r$-range resolution of SAR data, $\theta$-incidence angle, $\lambda$ - wavelength, $R_{S}$ - range distance between SAR sensors and earth surface. Reasons of appearing a low coherence and decorrelation $\gamma_{\text {temp }}$ is many, they often found on areas covered by vegetation, especially forests, lose coherence within a few days, especially due to wind and in the long-term also due to plant growth.

Hosokawa et al. proposed temporal coherence ratio for detection of building damage [20]

$$
\eta_{t}=\frac{\rho_{t}^{23}}{\rho_{t}^{12}}=\frac{\gamma^{(23)}}{\gamma^{(12)}} \cdot \frac{\rho_{n}^{12}}{\rho_{n}^{23}}
$$

where $\rho_{n}^{12} \approx \rho_{n}^{23}$, then, $\gamma^{(23)}-$ coseismic coherence coefficient and $\gamma^{(12)}$ pre-earthquake coherence coefficient. Theoretical probability of distribution temporal coherence ratio range $\eta_{0}$ is $0.6 \div 0.7$. Coherence ratio value for different type of damaged building shown in damage estimation model. It is displayed via cumulative probability of damage degree $(\%)$ and temporal coherence ratio $\eta_{t}$, here it is consist four classes serious damaged, major damaged, minor damaged, undamaged [19].

Yonezawa et al. proposed method based on decorrelation coherence coefficient for that they used normalized difference value for estimation of damaged building in urban area [16]. The normalized difference calculation is same like for intensity correlation (3), which was mentioned before:

$$
N d_{c}=\left[\frac{\rho_{c_{1}}-\rho_{c_{2}}}{\rho_{c_{1}}+\rho_{c_{2}}}\right] \times G n+O f
$$

where $\rho_{c_{1}}$ and $\rho_{c_{2}}$ are coherence coefficients before earthquake and coseismic respectively.

Hoffman suggested the same temporal coherence ratio only contrary [22]:

$$
\rho=\frac{\gamma_{\text {before }}}{\gamma_{\text {coseismic }}}
$$

where $\gamma_{\text {before }}$ and $\gamma_{\text {coseismic }}$ are coherence coefficients before earthquake and coseismic respectively. Here, this value was taken from 0 to 3 , and held hard classification at district (segment) level. See Table 1. The averaged coherence index was computed as

$$
\bar{\rho}=\frac{1}{N} \sum_{i=1}^{N} \min \left(\rho_{i}, 3\right)
$$

where $N$ - total number pixels in segment, all of value $\rho_{i}$ which will be summed should not be above 3 , if they exist then they are replaced by 3 . 
Table 1. Damage class definition [22]

\begin{tabular}{cc}
\hline Average coherence index range & Damage level class \\
\hline$\rho<1.5$ & No damage \\
$1.5 \leq \rho<2.0$ & Light damage \\
$2.0 \leq \rho<2.5$ & Significant damage \\
$\rho \geq 2.5$ & Severe damage \\
\hline
\end{tabular}

Wang et al. have proposed the use of Getis statistical and K-distribution method for building damage level with multi-temporal radar imageries [23,24]. This work based on simulated imageries, here, some parameters Kdistribution from this simulated image and the standardized version of Getis statistics are calculated:

$$
\begin{gathered}
Z_{i}(d)=\frac{\left[G_{i}(d)-E\left(G_{i}^{*}(d)\right)\right]}{\sqrt{\operatorname{Var}\left(G_{i}^{*}(d)\right)}} \\
G_{i}^{*}=\sum_{j=1}^{n} \frac{W_{i j}(d) x_{j}}{\sum_{j=1}^{n} x_{j}}
\end{gathered}
$$

where $G_{i}^{*}$ - Getis statistics for one $n$ - pixel image, the ratio of pixels centered on the $i$ - th pixel within distance $\mathrm{d}$ and the pixels of entire image, $W_{i j}$ is a weighting matrix, which takes " 1 " for the pixel with distance less than $d$ from pixel $i$, and zero otherwise, $E(\cdot)$ denotes the mean value and $\operatorname{Var}(\cdot)$ means variance. These formulas were used for pre- and post-earthquake ALOS PALSAR imageries over Beichuan city to detect of changing, by using difference of $Z$ images [23,25].

Seismic intensity with its integration with existing data first demonstrated Matsuoka et al. [26], there was defined the relationship between the discriminant score $\mathrm{Z}_{\mathrm{Rj}}$ and the JMA (Japan Meteorological Agency) seismic intensity in this work. See Fig. 1.

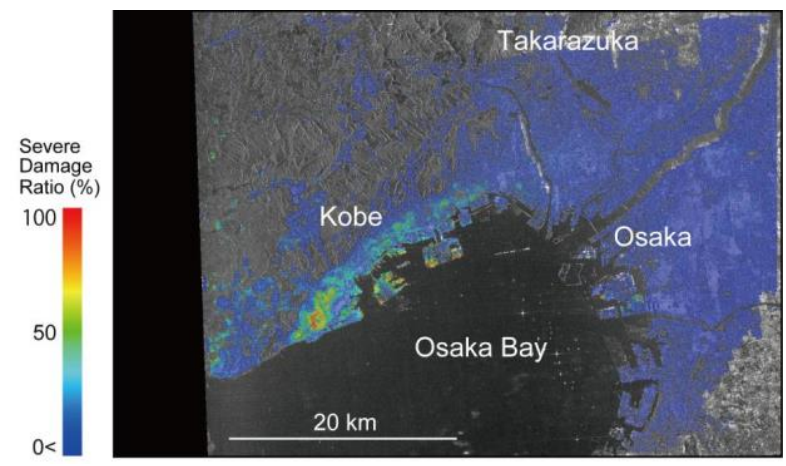

Figure 1. Distribution of estimated severe damage ratios (average values), estimated from an integrated processing of the discriminant score $Z_{R j}$ of the JERS-1 radar imageries and seismic intensity information [18]

All presented works have been associated with the use of low resolution SAR data, then appeared high-resolution SAR data, such as TerraSAR-X, Radarsat-2, COSMOSkyMed and etc., which are able to see individual buildings and homes. Guida et al. proposed a method based on double-bounce changing using Cosmo Skymed data, however, this research is true for single buildings [2]. Uprety and Yamazaki used multi-temporal TerraSAR-X and high resolution multispectral data, such as Quickbird,
GeoEye-1, which are used to generate intensity correlation coefficient, backscattering coefficients, their difference and NDVI values respectively [26]. Early, Stramondo and Bignami combined the capability of detecting earthquake damages in urban areas using ERS-1, ENVISAT ASAR, IRS1-C, TERRA ASTER. They used intensity correlation, interferometric coherence and NDVI also (masking vegetation) for building damage estimation [27]. Chini et al. had been used many optical data with multi-temporal ENVISAR ASAR data, which include near and termal infrared band for assessment of Tohoku tsunami consequences, such as inundation, liquefaction and washing away effects [28]. In subsequent studies, there have been used GIS data, which include not only GIS layers with city parcel boundaries [10], but also individual information, such as shape, size, high of building [29].

Overall accuracies of damage assessment were obtained Stramondo et al. in low resolution radar remote sensing data and their combination with optical data [34], [35]. It showed maximum overall accuracy with interferometric coherences (pre-seismic, co-seismic, postseismic) and optical (pre- and post-seismic) data. See Fig. 2 .

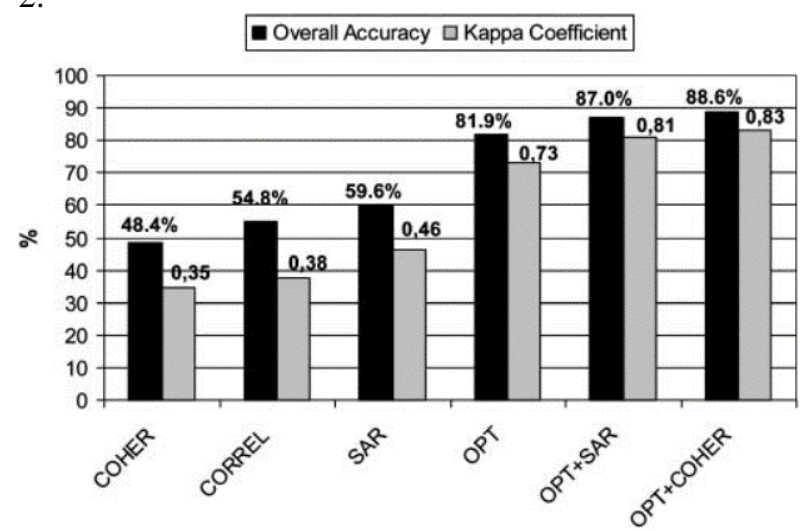

Figure 2. Results of pixel-by-pixel classification of damaged areas in terms of overall accuracy and Kappa coefficient with six classes: settlement build after earthquake, building with decreased dimension (probably collapsed), rubbles, subsidence, damaged (burnt) oil tanks, unchanged urban area (Stramondo 2007) [34]

Overall accuracy of damage assessment becomes higher when there have more data (including different sensors). High resolution radar imageries give higher overall accuracy than low resolution data, for example, it is greater (by 10 - 13 per cent) with combination of the difference of backscattering and intensity correlation coefficients for TerraSAR-X [26]. Overall accuracy is higher when spatial resolution of radar remote sensing data is higher.

Karimzadeh et. al. have used these combinations in their scientific works for detection of damaged urban area in Amatrice town struck by the Central Italy Earthquake, 2016 [31]. They have used these calculated values: mean differential intensity and mean differential coherence with discriminant score. Sentinel-1 and ALOS PALSAR 2 data were applied, result showed better accuracy with Sentinel1A/B. Ferrentino et. al. were proposed to use a coherent dual-polarimetric feature based on the inter-channel 
coherence [32], then Lagrange optimization of the difference between two polarimetric covariance matrices were offered in followed works [33].

\section{PROPOSED METHODOLOGY}

A multi-temporal backscattering coefficients and coherence coefficients (pre-event and post-event) coregistered dataset was used in new proposed method with GLCM texture parameters [40-42]. The block diagram of this method is shown below. See Fig. 3. The satellite dataset was selected of three Sentinel-1A/B imageries (pre- and post- events data) from descending orbits for Amatrice town struck by Italy Earthquake on 24 August 2016. They are shown on Table 2.

\begin{tabular}{clc}
\multicolumn{3}{c}{ Table 2. Satellite data } \\
\hline Satellite & \multicolumn{1}{c}{ Acquisition date } & $\begin{array}{c}\text { Ground } \\
\text { resolution }\end{array}$ \\
\hline Sentinel-1A & Pre-seismic 9 August 2016 & $13.89 \mathrm{~m} \times 3.37 \mathrm{~m}$ \\
& Pre-seismic 21 August 2016 & Single look complex \\
& Post-seismic 2 September 2016 & $($ az $\times$ rg)
\end{tabular}



Figure 3. Block diagram of new proposed method

All of the coregistered SAR values such as pre-seismic backscattering coefficient for dual polarizations $\sigma_{\text {pre }}^{V V}$, $\sigma_{\text {pre }}^{V H}$, post-seismic $\sigma_{\text {post }}^{V V}, \sigma_{\text {post }}^{V H}$ and pre-seismic coherence coefficients $\rho_{\text {pre }}$, coseismic $\rho_{\text {cos }}$ are used further for texture image processing.

To assess the degree of change, the ratio of pre- and post-seismic backscattering coefficients were computed for dual polarizations:

$$
\eta_{V V}=\frac{\sigma_{\text {pre }}^{V V}}{\sigma_{\text {post }}^{V V}}, \quad \eta_{V H}=\frac{\sigma_{\text {pre }}^{V H}}{\sigma_{\text {post }}^{V H}}
$$

Gray Level Co-ocurrence Matrix (GLCM) is most common used for calculation of textural features, first time Haralick et al. have been proposed to use it for image classification [41]. GLCM has window size and eight textural features: mean, variance, second moment, homogeneity, correlation, dissimilarity, entropy, contrast. Next GLCM parameters are better for for detection of damaged building, because there are comparable better separability between damaged and intact buildings cases:

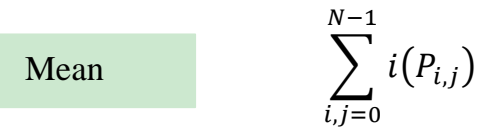

$\begin{array}{ll}\text { Homogeneity } & \sum_{\substack{i, j=0 \\ N-1}}^{N-1} \frac{P_{i, j}}{1+(i-j)^{2}} \\ \text { Dissimilarity } & \sum_{i, j=0}^{N-1} P_{i . j}|i-j|\end{array}$

\section{RESULT ANALYSIS}

Simultaneous use of different GLCM textural parameters for Sentinel-1 radar imageries with VV and VH polarization channels gave the following results on the distribution graph of ratio of backscattering coefficients $\sigma_{\text {pre }}^{V V} / \sigma_{\text {post }}^{V V}, \sigma_{\text {pre }}^{V H} / \sigma_{\text {post }}^{V H}$. Homogeneity and Dissimilarity GLCM parameters in $5 \times 5$ window size showed good separation of intact and completely destroyed building than other textural parameters. See Fig. 4,5.

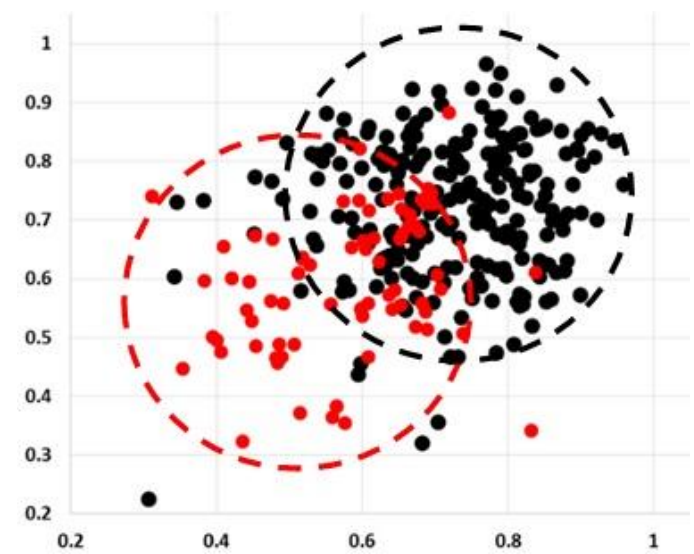

Figure 4. 2D-plot of Homogeneity GLCM $5 \times 5, \mathrm{x}-$ axis $\sigma_{\text {pre }}^{V V} / \sigma_{\text {post }}^{V V}, \mathrm{y}-$ axis $\sigma_{\text {pre }}^{V H} / \sigma_{\text {post }}^{V H}$ (Black point - intact building: 239 samples, Red - damaged building: 79 samples

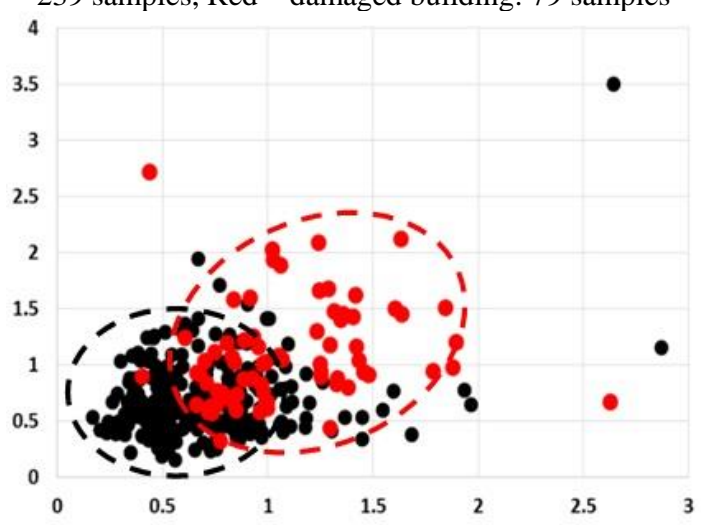

Figure 5. 2D-plot of Dissimilarity GLCM 5 $\times 5, \mathrm{x}-$ axis $\sigma_{\text {pre }}^{V V} / \sigma_{\text {post }}^{V V}, \mathrm{y}-$ axis $\sigma_{\text {pre }}^{V H} / \sigma_{\text {post }}^{V H}$ (Black point - intact building:

239 samples, Red - damaged building: 79 samples

Histogram of coherence coefficients ratio $\mathrm{VV}$ polarization $\rho_{\text {cos }}^{V V} / \rho_{\text {pre }}^{V V}$ of Mean GLCM parameters showed showed good separation of intact and damaged buildings in different damage level (moderately, highly and completely destroyed) in 0,65 threshold value than other textural parameters. The window size $3 \times 3$ for 
computation of the Mean GLCM textural parameter is the most optimal for separation than others. See Fig. 6.

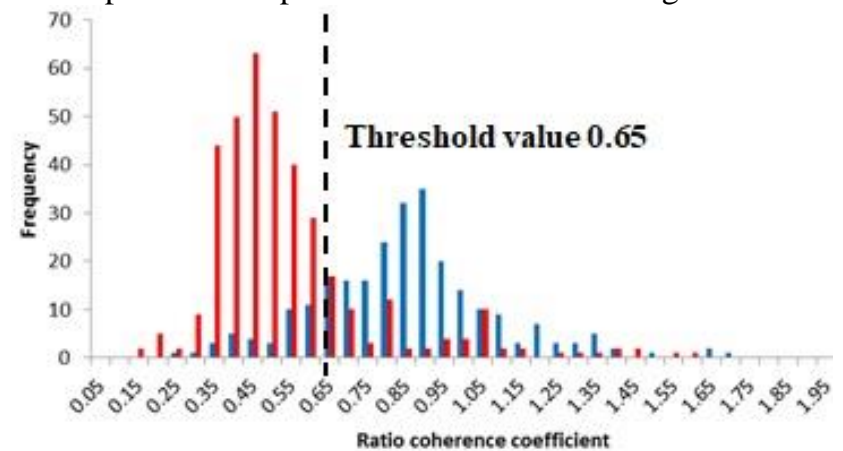

Figure 6. Histogram of mean GLCM $3 \times 3$ of ratio coherence coefficient $\rho_{\text {cos }}^{V V} / \rho_{\text {pre }}^{V V} \mathrm{VV}$ polarization (red damaged building, blue - intact building)

Mahalanobis and Maximum Likelihood supervised classification methods give good optimal results for building damage assessment using 4 and 6 different inputs (Table 3):

1) Homogeneity GLCM $5 \times 5-\sigma_{\text {pre }}^{V V} / \sigma_{\text {post }}^{V V}(\mathrm{HomVV})$

2) Homogeneity GLCM $5 \times 5-\sigma_{\text {pre }}^{V H} / \sigma_{\text {post }}^{V H}(\mathrm{HomVH})$

3) Dissimilarity GLCM $5 \times 5-\sigma_{\text {pre }}^{V V} / \sigma_{\text {post }}^{V V}($ DissVV)

4) Dissimilarity GLCM $5 \times 5-\sigma_{\text {pre }}^{V H} / \sigma_{\text {post }}^{V H}($ DissVH)

5) Mean GLCM $3 \times 3-\rho_{\text {cos }}^{V V}$ (Coh_cos)

6) Mean GLCM $3 \times 3-\rho_{\text {pre }}^{V V}$ (Coh_pre)

Table 3. Supervised classification results for new proposed method using GLCM textural parameters

\begin{tabular}{|c|c|c|c|c|}
\hline \multirow{2}{*}{} & \multicolumn{2}{|c|}{$\begin{array}{l}\text { HomVV, HomVH, DissVV, } \\
\text { DissVH, Coh_pre, Coh_cos }\end{array}$} & \multicolumn{2}{c|}{$\begin{array}{c}\text { HomVV, HomVH, } \\
\text { Coh_pre,Coh_cos }\end{array}$} \\
\cline { 2 - 5 } & $\begin{array}{c}\text { Maximum } \\
\text { Likelihood }\end{array}$ & Mahalanobis & $\begin{array}{l}\text { Maximum } \\
\text { Likelihood }\end{array}$ & Mahalanobis \\
\hline OA & $\underline{\mathbf{0 . 7 7 0 8}}$ & $\mathbf{0 . 7 6 0 8}$ & $\mathbf{0 . 7 5 9 7}$ & $\mathbf{0 . 7 4 9 7}$ \\
\hline IN & $\mathbf{0 . 5 4 4 4}$ & $\underline{\mathbf{0 . 6 4 0 9}}$ & $\mathbf{0 . 6 2 1 6}$ & $\mathbf{0 . 6 3 3 2}$ \\
\hline DM & $\underline{\mathbf{0 . 8 5 7 5}}$ & $\mathbf{0 . 8 2 5}$ & $\mathbf{0 . 8 5 2 1}$ & $\mathbf{0 . 8 1 7 2}$ \\
\hline NB & $\underline{\mathbf{0 . 8 4 3 5}}$ & $\mathbf{0 . 7 8 0 2}$ & $\mathbf{0 . 7 6 3 7}$ & $\mathbf{0 . 7 6 3 7}$ \\
\hline
\end{tabular}

Overall accuracy (OA), producer's accuracy of intact building (IN), damaged building (DM), none building (NB). Damaged building class is result of merging moderately, highly and completely destroyed building classes.

Result image processing to create damage are showed in below, it is consists of three class: a) none built-up area; b) intact building; 3) damaged building. See Fig. 7. Google Earth image and Damage map of west part of Amatrice town presented for comparison. See Fig. 8,9.

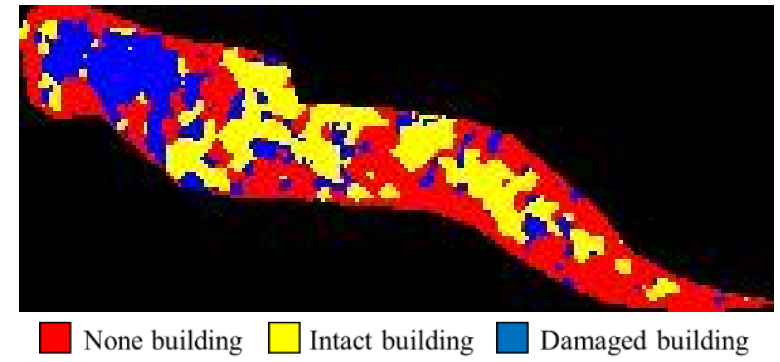

Figure 7. Mahalanobis distance supervised classification image with six inputs

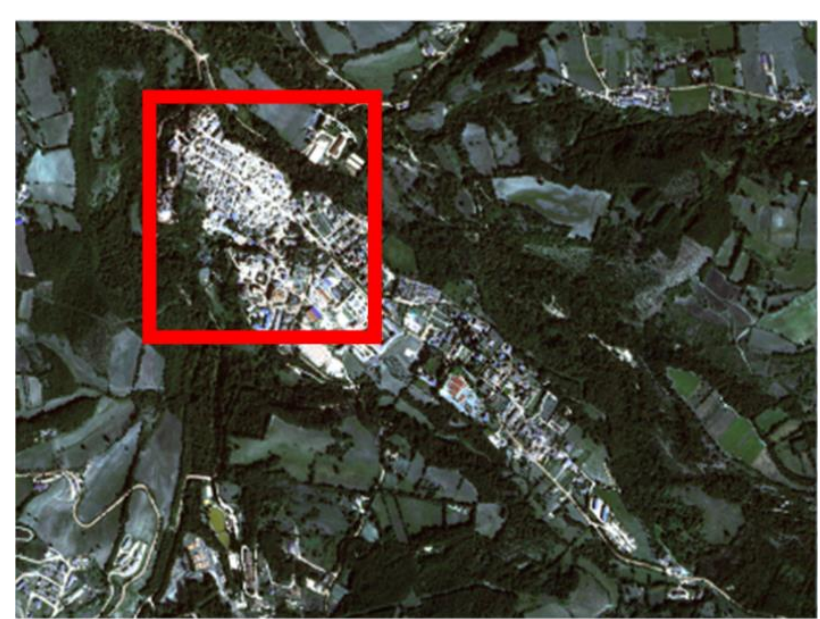

Study area is Amatrice town (red rectangular is more damaged part of town), Italy. Date: 2016-08-29

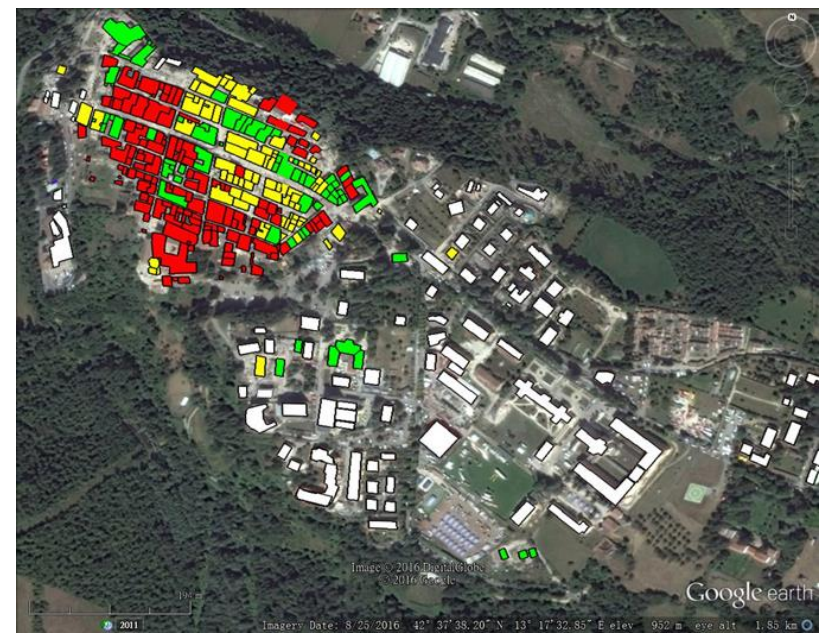

Figure 9. Google Earth image and Damage map (from Copernicus Emergency Management Service) of west part of Amatrice town. Legend: red - moderately, green - highly damaged and yellow - completely destroyed building

\section{CONCLUSION}

It is found that disadvantages of change detection method: a) large time between image acquisitions (especially for low-resolution radar imageries); b) required at least two acquisitions or more, multi-temporal data may not always be available (especially before event); c) more acquisitions means more costs (especially for high-resolution radar imageries). Advantages of change detection method: a) when all requirements are met related to acquisitions imageries (especially high resolution radar imageries), it is the most suitable to obtain high overall accuracy with the inclusion of all ancillary data and GIS data; b) method based on interferometric coherence enables better differentiation of slightly damaged and undamaged areas [36].

Low resolution Sentinel-1A/B data should be applied in future works for building damage assessment methods. It needs to focus on the use of textural features of interferometric coherence and intensity correlation 
coefficients for buildings damage assessment in future research [38].

A new proposed method using change detection method with GLCM texture parameters gives these conclusions. It was found that completely destroyed and intact buildings can be better distinguish by using GLCM parameters of backscattering coefficients $\left(\sigma_{\text {pre }}^{V V} / \sigma_{\text {post }}^{V V}, \sigma_{\text {pre }}^{V H} / \sigma_{\text {post }}^{V H}\right)$. Ratio of coherence coefficients $\sigma_{c o s}^{V V} / \sigma_{\text {pre }}^{V V}$ is sensitive to moderately damaged, highly damaged and completely destroyed buildings. It is very difficult to separate different classes of destruction of the buildings, for examples, moderately damaged and highly damaged or highly damaged and completely destroyed buildings. Producer's accuracy results are not sufficient for intact building class, because it is less than other classes (producer's accuracy is less than 0,64). Given the above insights, it is required to improve the method, in order to increase the producer's accuracy intact building GIS data of urban area is required, and also distinguish different level of damaged buildings.

Change detection methods require huge computational resources and advanced algorithm with additional GIS data. Because there is used for processing a pair of radar images with several tens of gigabytes of data. Radar image processing is time consuming, and this time depends of computing power. Parallel calculations are necessary to divide the radar image into smaller parts and each part is processed by its own processor. Currently, there are many solutions for image processing based on various mini PCs that can be combined into clusters, one of them may be based on the application of the large number of Raspberry Pi mini PCs [39].

\section{ACKNOWLEDGMENT}

The research leading to these results has received funding from the Ministry of Agriculture of the Republic of Kazakhstan under grant agreement number BR06349506. The author would like to thank European Space Agency for free access to Sentinel-1 radar remote sensing data.

\section{REFERENCES}

1. Brunner, D.; Lemoine, G.; Bruzzone, L. Earthquake Damage Assessment of Buildings Using VHR Optical and SAR Imagery. Ieee Transactions on Geoscience and Remote Sensing 2010, 48, 24032420, doi:10.1109/tgrs.2009.2038274.

2. Guida, R.; Iodice, A.; Riccio, D.; Ieee. MONITORING OF COLLAPSED BUILT-UP AREAS WITH HIGH RESOLUTION SAR IMAGES. 2010 Ieee International Geoscience and Remote Sensing Symposium 2010, 2422-2425, doi:10.1109/igarss.2010.5650083.

3. Matsuoka, M.; Yamazaki, F. Use of satellite SAR intensity imagery for detecting building areas damaged due to earthquakes. Earthquake Spectra 2004, 20, 975-994, doi:10.1193/1.1774182.
4. Brunner, D.; Lemoine, G.; Bruzzone, L.; Greidanus, H. Building Height Retrieval From VHR SAR Imagery Based on an Iterative Simulation and Matching Technique. Ieee Transactions on Geoscience and Remote Sensing 2010, 48, 1487 1504, doi:10.1109/tgrs.2009.2031910.

5. Balz, T.; Liao, M.S. Building-damage detection using post-seismic high-resolution SAR satellite data International Journal of Remote Sensing 2010, 31, 3369-3391, doi:10.1080/01431161003727671.

6. Yun, S.; Hua-ze, G.; Shi-ang, W.; Feng-li, Z.; Wei, T. Mulit-source SAR Remote Sensing Data for Rapid Response to Wenchuan Earthquake Damage Assessment. Journal of Remote Sensing 2008, 12, 865-870.

7. Wu, F.; Gong, L.X.; Wang, C.; Zhang, H.; Zhang, B.; Xie, L. Signature Analysis of Building Damage With TerraSAR-X New Staring SpotLight Mode Data. Ieee Geoscience and Remote Sensing Letters 2016, 13, 1696-1700, doi: 10.1109/lgrs.2016.2604841.

8. Bryan, M.L. URBAN LAND-USE CLASSIFICATION USING SYNTHETIC APERTURE RADAR. International Journal of Remote Sensing 1983, 4, 215-233.

9. Lee, J.S. SPECKLE ANALYSIS AND SMOOTHING OF SYNTHETIC APERTURE RADAR IMAGES. Computer Graphics and Image Processing 1981, 17, 24-32, doi:10.1016/s0146664x(81)80005-6.

10. Gamba, P.; Dell'Acqua, F.; Trianni, G. Rapid damage detection in the Bam area using multitemporal SAR and exploiting ancillary data. Ieee Transactions on Geoscience and Remote Sensing 2007, 45, 1582-1589, doi:10.1109/tgrs.2006.885392.

11. Jin, Y.-Q.; Wang, D. Automatic Detection of Terrain Surface Changes After Wenchuan Earthquake, May 2008, From ALOS SAR Images Using 2EM-MRF Method. Ieee Geoscience and Remote Sensing Letters 2009, 6, 344-348, doi:10.1109/lgrs.2009.2013875.

12. Yonezawa, C.; Takeuchi, S. Detection of urban damage using interferometric SAR decorrelation. In IEEE 1999 International Geoscience and Remote Sensing Symposium. IGARSS'99, Stein, T.I., Ed. Ieee: 1999; pp. 925-927 vol.922.

13. Matsuoka, M.; Yamazaki, F. Characteristics of satellite SAR images in the areas damaged by earthquakes. Igarss 2000: Ieee 2000 International Geoscience and Remote Sensing Symposium, Vol I Vi, Proceedings 2000, 2693-2696.

14. Hosokawa, M.; Jeong, B.P.; Takizawa, O.; Ieee. Earthquake damage detection using remote sensing data. Igarss: 2007 Ieee International Geoscience and Remote Sensing Symposium, Vols 1-12: Sensing and Understanding Our Planet 2007, 2989-+, doi:10.1109/igarss.2007.4423473.

15. Yonezawa, C.; Takeuchi, S. Decorrelation of SAR data by urban damages caused by the 1995 Hyogoken-nanbu earthquake. International Journal 
of Remote Sensing 2001, 22, 1585-1600, doi:10.1080/01431160118187.

16. Yonezawa, C.; Tomiyama, N.; Takeuchi, S.; Ieee. Urban damage detection using decorrelation of SAR interferometric data. Igarss 2002: Ieee International Geoscience and Remote Sensing Symposium and 24th Canadian Symposium on Remote Sensing, Vols I-Vi, Proceedings: Remote Sensing: Integrating Our View of the Planet 2002, 2051-2053.

17. Matsuoka, M.; Yamazaki, F. Building Damage Mapping of the 2003 Bam, Iran, Earthquake Using Envisat/ASAR Intensity Imagery. Earthquake Spectra 2005, 21, S285-S294, doi:10.1193/1.2101027.

18. Matsuoka, M.; Nojima, N. Building Damage Estimation by Integration of Seismic Intensity Information and Satellite L-band SAR Imagery. Remote Sensing 2010, 2, 2111-2126, doi:10.3390/rs2092111.

19. Ito, Y.; Hosokawa, M.; Matsuoka, M.; Ieee. A degree estimation model of earthquake damage using temporal coherence ratio. Igarss 2003: Ieee International Geoscience and Remote Sensing Symposium, Vols I - Vii, Proceedings: Learning from Earth's Shapes and Sizes 2003, 2410-2412.

20. Ito, Y.U.; Hosokawa, M.; Ieee. Damage estimation model using temporal coherence ratio. Igarss 2002: Ieee International Geoscience and Remote Sensing Symposium and 24th Canadian Symposium on Remote Sensing, Vols I-Vi, Proceedings: Remote Sensing: Integrating Our View of the Planet 2002, 2859-2861.

21. Strozzi, T.; Dammert, P.B.G.; Wegmuller, U.; Martinez, J.M.; Askne, J.I.H.; Beaudoin, A.; Hallikainen, M.T. Landuse mapping with ERS SAR interferometry. Ieee Transactions on Geoscience and Remote Sensing 2000, 38, 766-775, doi:10.1109/36.842005.

22. Hoffmann, J. Mapping damage during the Bam (Iran) earthquake using interferometric coherence. International Journal of Remote Sensing 2007, 28, 1199-1216, doi:10.1080/01431160600928567.

23. Haipeng, W.; Ya-Qiu, J. Statistical analysis to assess building damage in 2008 Wenchuan earthquake from multi-temporal SAR images. In Proceedings of the 2009 2nd Asian-Pacific Conference on Synthetic Aperture Radar, Wu, S., Ed. Ieee: 2009; pp. 121-123.

24. Getis, A.; Ord, J.K. THE ANALYSIS OF SPATIAL ASSOCIATION BY USE OF DISTANCE STATISTICS. Geographical Analysis 1992, 24, 189-206, doi:10.1111/j.1538-4632.1992.tb00261.x.

25. Wang, C.; Zhang, H.; Wu, F.; Zhang, B.; Tang, Y.X.; Wu, H.A.; Wen, X.Y.; Yan, D.M. Disaster phenomena of Wenchuan earthquake in high resolution airborne synthetic aperture radar images. Journal of Applied Remote Sensing 2009, 3, 16, doi:10.1117/1.3154558.

26. Uprety, P.; Yamazaki, F.; Ieee. USE OF HIGHRESOLUTION SAR INTENSITY IMAGES FOR DAMAGE DETECTION FROM THE 2010 HAITI
EARTHQUAKE. 2012 Ieee International Geoscience and Remote Sensing Symposium (Igarss) 2012, 6829-6832, doi:10.1109/igarss.2012.6352595.

27. Bignami, C.; Chini, M.; Pierdicca, N.; Stramondo, S.; ieee. Comparing and combining the capability of detecting earthquake damages in urban areas using SAR and optical data. Igarss 2004: Ieee International Geoscience and Remote Sensing Symposium Proceedings, Vols 1-7: Science for Society: Exploring and Managing a Changing Planet 2004, 55-58.

28. Chini, M.; Piscini, A.; Cinti, F.R.; Amici, S.; Nappi, R.; De Martini, P.M. The 2011 Tohoku (Japan) Tsunami Inundation and Liquefaction Investigated Through Optical, Thermal, and SAR Data. Ieee Geoscience and Remote Sensing Letters 2013, 10, 347-351, doi:10.1109/lgrs.2012.2205661.

29. Mansouri, B.; Shinozuka, M.; Huyck, C.; Houshmand, B. Earthquake-Induced Change Detection in the 2003 Bam, Iran, Earthquake by Complex Analysis Using Envisat ASAR Data. Earthquake Spectra 2005, 21, S275-S284, doi:10.1193/1.2098987.

30. Takeuchi, S.; Suga, Y.; Yonezawa, C.; Chen, A.J. Detection of urban disaster using InSAR - A case study for the 1999 Great Taiwan Earthquake. Igarss 2000: Ieee 2000 International Geoscience and Remote Sensing Symposium, Vol I - Vi, Proceedings 2000, 339-341.

31. Karimzadeh, S.; Mastuoka, M. Building Damage Assessment Using Multisensor Dual-Polarized Synthetic Aperture Radar Data for the 2016 M 6.2 Amatrice Earthquake, Italy. Remote Sensing 2017, 9 , 17, doi:10.3390/rs9040330.

32. Ferrentino, E.; Nunziata, F.; Migliaccio, M.; Vicari, A. A sensitivity analysis of dual-polarization features to damage due to the 2016 Central-Italy earthquake. International Journal of Remote Sensing 2018, 39, 6846-6863, doi:10.1080/01431161.2018.1466078.

33. Ferrentino, E.; Marino, A.; Nunziata, F.; Migliaccio, M. A dual-polarimetric approach to earthquake damage assessment. International Journal of Remote Sensing 2019, 40, 197-217, doi:10.1080/01431161.2018.1511935.

34. Stramondo, S.; Bignami, C.; Chini, M.; Pierdicca, N.; Tertulliani, A. Satellite radar and optical remote sensing for earthquake damage detection: results from different case studies. International Journal of Remote Sensing 2006, 27, 4433-4447, doi:10.1080/01431160600675895.

35. Stramondo, S.; Bignami, C.; Pierdicca, N.; Chini, M. SAR and optical remote sensing for urban damage detection and mapping: case studies. In 2007 Urban Remote Sensing Joint Event, Ieee: 2007; pp. 704-709.

36. Plank, S. Rapid Damage Assessment by Means of Multi-Temporal SAR - A Comprehensive Review and Outlook to Sentinel-1. Remote Sensing 2014, 6, 4870-4906, doi:10.3390/rs6064870. 
37. Christakis, Ch., Ioannidi, K., Sautbekov, S., Frangos, P., Atanov, S.K. The radiation problem from a vertical short dipole antenna above flat and lossy ground: Novel formulation in the spectral domain with closed -form analytical solution in the high frequency regime. Elektronika ir Elektrotechnika 2014, Vol.20, №9, 35-38, doi: https://doi.org/10.5755/j01.eee.20.9.8710

38. Asset Akhmadiya, Qiming Zeng. Use of Sentinel-1 Data for Earthquake Damage Assessment in Cases of Amatrice and Sarpol-E Zahab. 2018 IEEE International Geoscience and Remote Sensing Symposium. doi: 10.1109/IGARSS.2018.8517285

39. Brimzhanova S. S., Atanov S. K., Moldamurat Khuralay, Kobelekov K. S., Gagarina L. G. Crossplatform compilation of programming language Golang for raspberry pi. ICEMIS '19: Proceedings of the 5th International Conference on Engineering and MIS, June 2019, Pages 1-5, doi: https://doi.org/10.1145/3330431.3330441

40. Ling-jun, Z.; Yu-liang, Q.I.N.; Gui, G.A.O.; Gangyao, K. Detection of built-up areas from highresolution SAR images using the GLCM textural analysis. Journal of Remote Sensing 2009, 13, 475490.

41. Haralick, R.M.; Shanmugam, K.; Dinstein, I. TEXTURAL FEATURES FOR IMAGE CLASSIFICATION. Ieee Transactions on Systems Man and Cybernetics 1973, SMC3, 610-621, doi:10.1109/tsmc.1973.4309314.

42. Ulaby, F.T.; Kouyate, F.; Brisco, B.; Williams, T.H.L. TEXTURAL INFORMATION IN SAR IMAGES. Ieee Transactions on Geoscience and Remote Sensing 1986, 24, 235-245, doi:10.1109/tgrs.1986.289643. 